\title{
Random distributed feedback Raman fiber laser with polarized pumping
}

\author{
H. Wu ${ }^{1}$, Z. N. Wang ${ }^{1}$, D. V. Churkin ${ }^{2,3,4}$, I. D. Vatnik ${ }^{2,3}$, M. Q. Fan ${ }^{1}$, and Y. J. Rao ${ }^{1}$ \\ ${ }^{1}$ Key Lab of Optical Fiber Sensing \& Communications, University of Electronic Science \&Technology of \\ China, Chengdu, Sichuan, China 611731 \\ ${ }^{2}$ Institute of Automation and Electrometry SB RAS, Novosibirsk 630090, Russia \\ ${ }^{3}$ Novosibirsk State University, Novosibirsk 630090, Russia \\ ${ }^{4}$ Aston Institute of Photonic Technologies, Aston University, Birmingham, B4 7ET, UK \\ Email: znwang@uestc.edu.cn
}

\begin{abstract}
In this paper, the polarization properties of random fiber laser operating via Raman gain and random distributed feedback owing to Rayleigh scattering is investigated for the first time. Using polarized pump, the partially polarized generation is obtained with a generation spectrum exhibiting descrete narrow spectral features contrary to the smooth spectrum observed for the unpolarized pump. The threshold, output power, degree of polarization (DOP) and the state of polarization (SOP) of the lasing can be significantly influenced by the SOP of the pump. Fine narrow spectral components are also sensitive to the SOP of the pump wave. Furthermore, we found that random lasing's longitudinal power distribution is different in the case of polarized and unpolarized pumpoing that results in considerable reduction of the generation slope efficiency for the polarized radiation. Our results indicates that polarization effects plays an important role in the performance of the random fiber laser. This work improves the understanding of the physics of random lasing in fibers and makes a step forward towards the establishment of the vector model of random fiber lasers.
\end{abstract}

Keywords: random laser, Raman laser, random distributed feedback laser

\section{Introduction}

Random laser (RL) refers to a new kind of lasers where the feedback is provided by randomly distributed scattering centers in a gain medium [1]. RL has been demonstrated in a wide range of random mediums, emission wavelengths, and scattering regimes [2-5]. Meanwhile, a number of potential applications of random lasers have been reported, including biomedical imaging, distributed amplification, remote sensing and display technology [6-11].

As an important type of RLs, random fiber laser via Raman gain and Rayleigh scattering along single mode fiber (SMF) as the random distributed feedback has been demonstrated since $2010[12,13]$. Various aspects of RFL have been studied in recent years. RFL has demonstrated to be tunable[14], multi-wavelength[15-18], narrow bandwidth[19], cascaded operation to generate high order Stokes wave[20-23] and high output power[24,25].

However, up till now, there are still a lot of open questions regarding the physics of random lasing in fibers. One of the important laser characteristics is the polarization characteristic.
The emission polarization of $\mathrm{RL}$ has been investigated in several random mediums, such as two-dimensional rod array [26], organic dye solutions [27,28] and dye-doped nematic liquid crystal (NLC) system [29]. In the conventional Raman fiber laser, the Raman gain is highly depended on the polarization [30]. It is also found that the dynamical behavior of the laser is depended on the states of polarization of the Stokes and pump fields and the fiber type (standard single-mode fiber or the polarization-maintaining fiber) [31]. Referring to the RFL, since the simulated Raman scattering (SRS), simulated Brillouin scattering (SBS) and four-wave-mixing (FWM) in fiber are highly dependent on polarization, the polarization properties of random lasing emission in such one-dimensional medium are important. However, in the previous work, the polarization properties of RFL have not been explored yet.

In this paper, we focus on the RFL via Raman gain and Rayleigh feedback, and study its polarization properties utilizing polarized pump and non-polarization-maintaining fiber. It is found that the random lasing shows the 
unique narrow spectral features with the polarized pumping. And the lasing threshold, the lasing spectral shape, the output lasing power, the state of polarization (SOP) and degree of polarization (DOP) of the lasing all vary substantially with SOP of the pump. Moreover, with the polarized pumping, due to the different average gain coefficient for forward and backward Stokes waves, the random lasing's power distribution is changed and the slope efficiency of pump side output is significantly reduced comparing to the depolarized pumping.

\section{Experimental setup}

The experimental setup is depicted in Fig. 1. Utilizing Stokes description, the Stokes parameters of a light are given by $S=\left(S_{0}, S_{1}, S_{2}, S_{3}\right)$, and the DOP of the light is defined by $D O P=\frac{\sqrt{S_{1}^{2}+S_{2}^{2}+S_{3}^{2}}}{S_{0}} \mathrm{gl} 00 \%$ pump is a depolarized fiber laser source whose degree of polarization (DOP) is measured as $\sim 5 \%$. To investigate the polarization properties of the random fiber laser, we insert a polarizer to make the $1365 \mathrm{~nm}$ pump polarized. The polarization controller (PC) is used to adjust the state of polarization (SOP) of $1365 \mathrm{~nm}$ pump before injecting into the $50 \mathrm{~km}$ SMF. The $1455 \mathrm{~nm}$ random lasing towards the pump side is extracted from the $1365 / 1455 \mathrm{~nm}$ WDM and split the $1 / 99$ coupler, and the $1 \%$ output is further split by the 50/50 coupler. The output ports $\mathrm{A}, \mathrm{B}$ and $\mathrm{C}$ are used for monitoring output power, optical spectrum and output polarization properties simultaneously. We also measure the laser output properties at the far end of $50 \mathrm{~km}$ SMF (Port D). It should be noted that all the fiber ends are angle-cleaved. We also checked the reflectivity of the angle-cleaved fiber end used in experiment is in the range of $10^{-4}$ to $10^{-5}$.

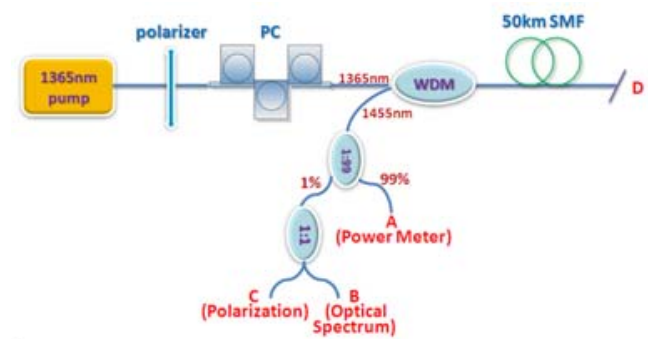

Fig.1. Experimental setup for RFL with polarized pump.

\section{Results and discussions}

Firstly, we fix a PC state and increase the pump power to excite the $1455 \mathrm{~nm}$ random lasing. The spectra evolution (observed at port B) with the pump power (the actual power launched into the fiber) is shown in Fig. 2. When the pump power is below the threshold, only the broad Raman gain curve exists. Then some discrete peaks appear in the broadband spontaneous spectrum when pump power is beyond $1.2 \mathrm{~W}$. When the pump power is increased to $1.27 \mathrm{~W}$, a narrow peak stimulated and its intensity increases rapidly. And further increase the pump power, there are more sharp peaks in the spectrum.

It should be noted that the spectra evolution of random lasing with polarized pump is quite different from the previous case with depolarized pump [10].The chaos regime which has many random spikes on the spectrum has not been observed. Moreover, the stable spectrum has several discrete narrow peaks. These narrow spectral components are new observations in the field of random fiber lasers. In the previous works with the depolarized pumping, the stable spectrum is always smooth.

The only realization of a random fiber laser with a fine-structure spectrum was reported in [Vatnik, I. D., Churkin, D. V., Babin, S. A. \& Turitsyn, S. K. Cascaded random distributed feedback Raman fiber laser operating at $1.2 \mu \mathrm{m}$. Opt. Express 19, 18486-94 (2011).], yet the nature of the structures was left unconsidered, and might be due to specific scheme employing pump laser inside the random cavity.

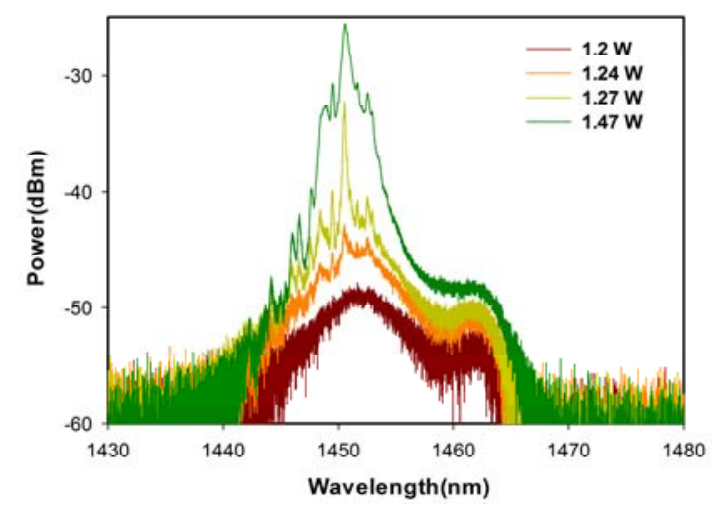

Fig. 2. Spectra evolution with polarized pump.

Furthermore, when we rotate the $\mathrm{PC}$ and thus change the SOP of injected pump, the random lasing's spectrum, output power and the 
degree of polarization (DOP) all change drastically. Fig.3 and Fig.4 show the spectra (record at port B), output power, DOP and SOP (record at port $\mathrm{C}$ ) under different PC states at pump power $1.3 \mathrm{~W}$ and $1.47 \mathrm{~W}$, respectively. From Fig.3(a), we can clearly see the SOP of pump can alter the threshold and the excited state of random lasing. Fig.3(b) shows the SOP of the generated light wave, the three points on the Poincare sphere corresponding to the three aforementioned spectrum states in Fig.3(a).
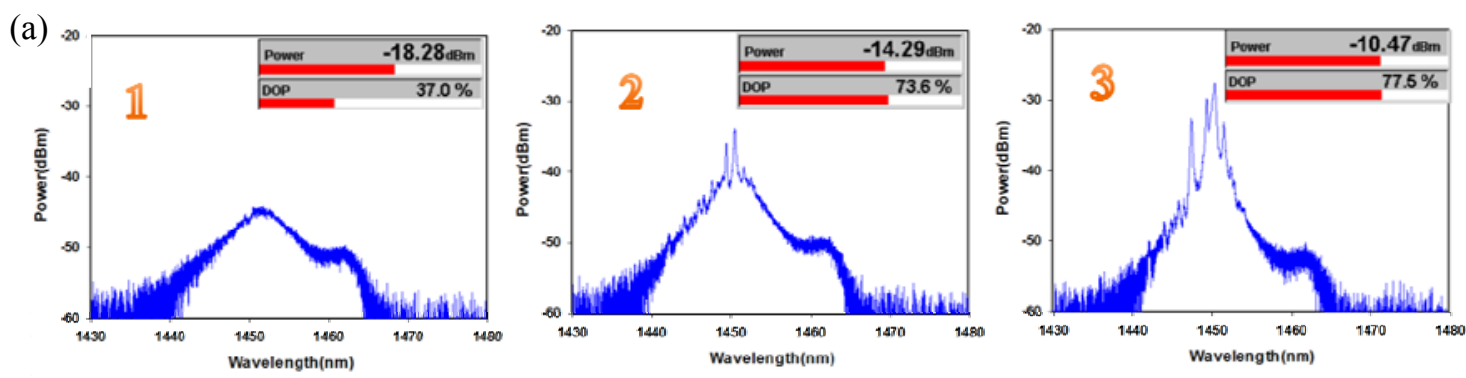

(b)

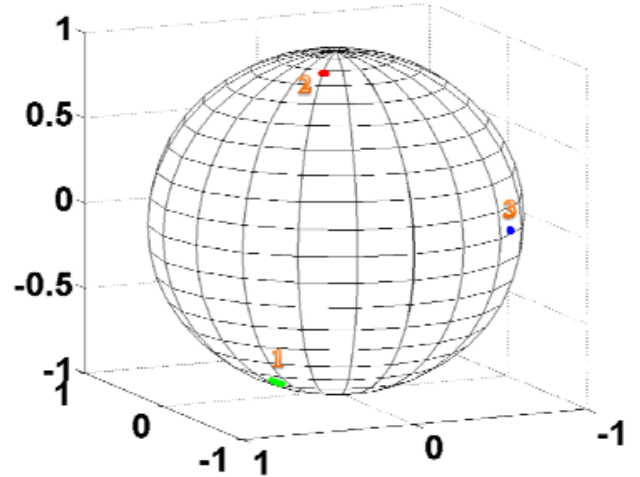

Fig. 3. Random lasing's properties under different PC states with $1.3 \mathrm{~W}$ pump power. (a) spectra, output power and DOP of the random lasing (b) SOP of the output random lasing
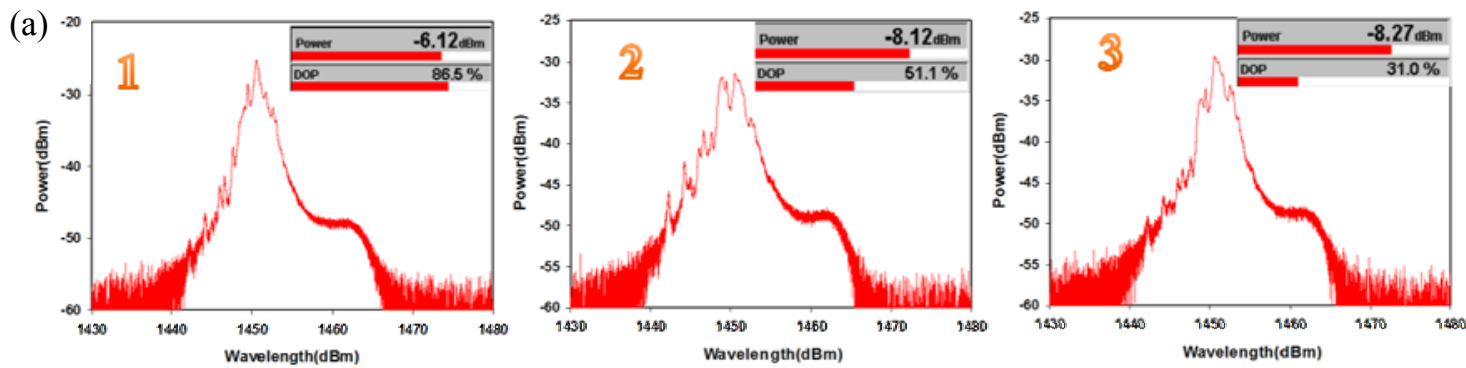
(b)

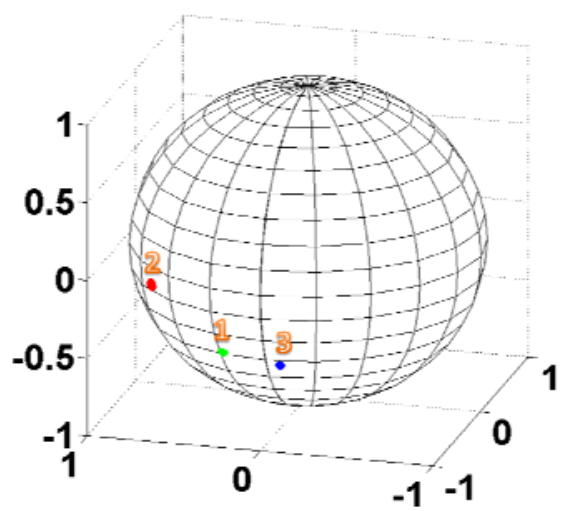

Fig. 4. Random lasing's properties under different PC states with $1.47 \mathrm{~W}$ pump power. (a) spectra, output power and DOP of the random lasing (b) SOP of the random lasing

When the random lasing is stimulated, the SOP of the output lasing is converged to a stable point on the Poincare sphere and also change with the SOP of the pump. In Fig.4, the pump power is well above the threshold in all SOP state of the pump, the random lasing is all stimulated with the different pump SOP, the PC state can change the lasing spectrum's shape, with the different narrow spectral features. The result can also indicate polarization plays an important role in formation of these narrow spectral components. Besides, the output power, DOP and SOP also vary substantially with the PC.

Since the output lasing power is varying with PC state, we adjust the PC state and record the output lasing powers at both the pump outputs of the laser: near the pump wave coupling point (port A) and at far end of the fiber (port D, through the WDM) in the case of high pump side output $\operatorname{DOP}(\sim 85 \%)$ and low pump side output DOP $(\sim 35 \%)$, respectively. In both cases, with various pump powers, the DOP varies less than $10 \%$. The results are shown in Fig.5 and Fig. 6. As a comparison, the output power vs pump power curve is also measured when the polarizer is removed (Fig.7). The thresholds for high DOP case and low DOP case are $1.2 \mathrm{~W}$ and $1.3 \mathrm{~W}$, respectively, which are all lower than the threshold $(1.5 \mathrm{~W})$ with depolarized pump. In the case of depolarized pump, since the power of random lasing mainly towards the pump side, the output power achieved at the pump side is very high and has a slope efficiency more than $60 \%$; Meanwhile, the output power from the fiber far-end is quite low and increases very slowly with the pump power, resulting in the power difference between the pump side and far-end increasing from $6.5 \mathrm{~dB}$ to $10 \mathrm{~dB}$. However, when the pump is polarized, the pump side's slope efficiency is much lower (less than 30\%). But it should be noted that the power difference between the pump side and far-end is also relatively low and kept nearly as a constant $(\sim 3 \mathrm{~dB})$. Therefore, it can be inferred that with the polarized pumping, the random lasing's power distribution is changed and more power is gathered in the interior of fiber. The power distribution difference between polarized and depolarized pump can be explained as follows: The Raman gain coefficient is highly dependent on the SOP of the pump and the Stokes wave, the Raman gain for the copolarized pump and Stokes waves is about two times larger than that for depolarized pump. Using the polarized pumping, the random low birefringence in single mode fiber will result in discrepancy between the gain coefficients of the forward and the backward Stokes wave, as the forward Stokes wave has the polarization evolution almost the same as the pump wave, and the backward Stokes wave changes the relative polarization rapidly and thus the gain coefficient of backward Stokes wave decreases [32]. As the result, by changing the average gain coefficient for forward and backward Stokes waves $\left(g_{f}\right.$ and $\left.g_{b}\right)$ correspondingly, we can qualitatively study the impact of polarization evolution on lasing power properties. 


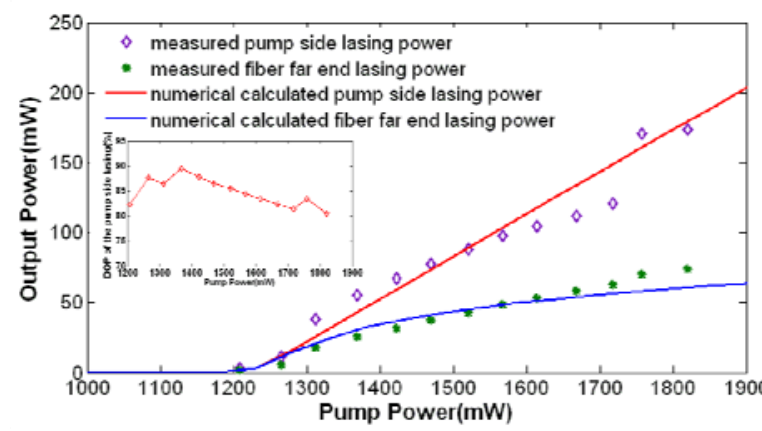

Fig. 5. Output power vs. pump power (high pump side DOP case) Insert: DOP variation with the pump power.

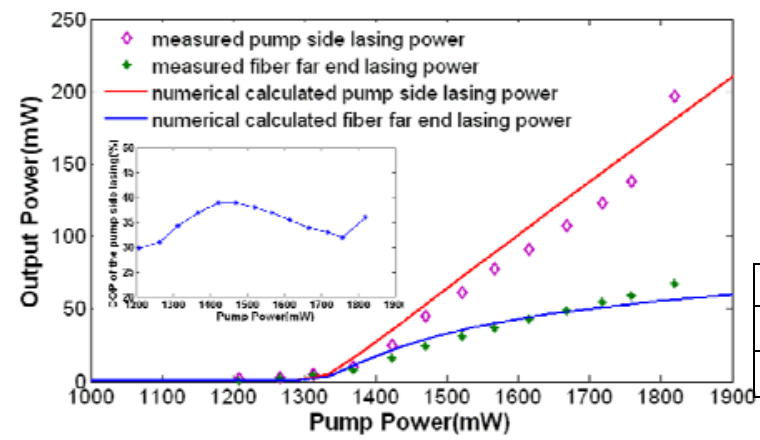

Fig. 6. Output power vs. pump power (low pump side DOP case) Insert: DOP variation with the pump power.

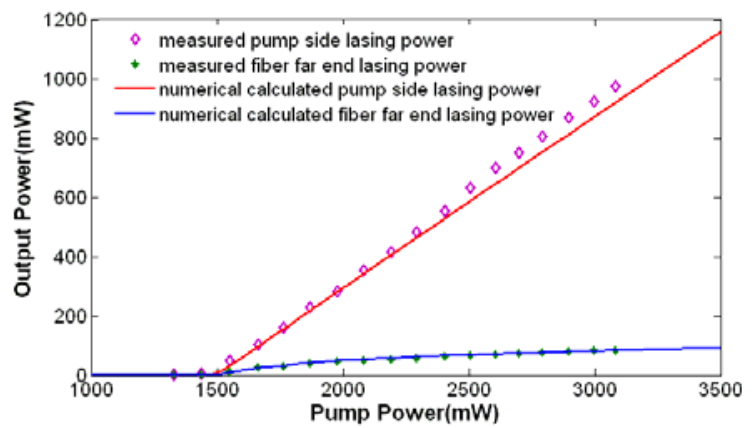

Fig. 7 output power vs pump power (without polarizer)

The modified steady-state power balance model can be written as [33]:

$\frac{d P_{0}^{+}}{d z}=-\alpha_{0} P_{0}^{+}-g_{f} \frac{f_{0}}{f_{1}} P_{0}^{+}\left(P_{1}^{+}+P_{1}^{-}+\Gamma_{1}\right)+\varepsilon_{0} P_{0}^{-}$

$\frac{d P_{0}^{-}}{d z}=\alpha_{0} P_{0}^{ \pm}+g_{b} \frac{f_{0}}{f_{1}} P_{0}^{-}\left(P_{1}^{+}+P_{1}^{-}+\Gamma_{1}\right)-\varepsilon_{0} P_{0}^{+}$

$\frac{d P_{1}^{+}}{d z}=-\alpha_{1} P_{1}^{ \pm}+g_{f}\left[P_{1}^{+}+0.5 \Gamma_{1}\right]\left(P_{0}^{+}+P_{0}^{-}\right)+\varepsilon_{1} P_{1}^{-}$

$\frac{d P_{1}^{-}}{d z}=\alpha_{1} P_{1}^{-}-g_{b}\left[P_{1}^{-}+0.5 \Gamma_{2}\right]\left(P_{0}^{+}+P_{0}^{-}\right)-\varepsilon_{1} P_{1}^{+}$

$\Gamma_{1}=4 h f_{1} \Delta f_{1}\left\{1+\frac{1}{\exp \left[h\left(f_{0}-f_{1}\right) /\left(K_{B} T\right)\right]-1}\right\}$ where lower indexes ' 0 ', ' 1 ' correspond to the pump, the 1st-order lasing, respectively. Lower indexes ' + ' and ' - ' denote the forward and backward waves, respectively. $P_{0,1}$, denotes the optical power, $f_{0,1}$ is the wave frequency. $\Gamma_{1}$ denotes the population of phonon, where $\Delta f_{1}=$ $0.25 \mathrm{THz}$ is the lasing bandwidth, $T(=298 \mathrm{~K})$ is the absolute temperature and $K_{B}$ is the Boltzmann's constant, $h$ is the Plank's constant, $\alpha_{0,1}$ is the fiber loss, $g_{f, b}$ is the Raman gain coefficients for forward and backward Stokes wave, $\varepsilon_{0,1}$ is the Rayleigh backscattering coefficient. The boundary conditions are $\quad P_{0}^{+}(0)=P_{\text {in }} \quad, \quad P_{1}^{+}(0)=R_{L} P_{1}^{-}(0)$ and $P_{1}^{-}(L)=R_{F} P_{1}^{+}(L)$ where $P_{\text {in }}$ denotes the pump power. The parameters used are summarized in Table I .

TABLE I

Parameters of the Fiber under Numerical calculation

\begin{tabular}{|c|c|c|}
\hline Wavelength(nm) & $\boldsymbol{\alpha}(\mathbf{d B} / \mathbf{k m})$ & $\boldsymbol{\varepsilon}\left(\mathbf{k m}^{-1}\right)$ \\
\hline 1365 & 0.32 & $1 \times 10^{-4}$ \\
\hline 1455 & 0.25 & $6 \times 10^{-5}$ \\
\hline
\end{tabular}

Under the condition of depolarized pumping, the gain coefficient $g_{f}$ and $g_{b}$ should be the same (0.50). However, when the pump is polarized, as discussed before, $g_{f}$ should be larger than $g_{b}$ and the $g_{f}$ and $g_{b}$ also vary with the SOP of the pump. As an example, we choose $g_{b}=0.54, g_{f}=0.65$ and Fig. 8 shows the lasing power distribution in this case. It is found that due to the higher gain coefficient for forward Stokes wave, more lasing power is distributed toward the far end of the fiber and meanwhile the output power at pump side is lower than that with depolarized pumping. To be consistent with the experimental data in Fig.4-6, we modify the $g_{f}$ and $g_{b}$ to account for the impact of polarization evolution and add the tiny parasitic reflection on the fiber end $\left(\mathrm{R}_{\mathrm{L}}=\mathrm{R}_{\mathrm{F}}=2 \times 10^{-4}\right)$. Table II gives the values of $g_{f}$ and $g_{b}$ for three cases in the numerical simulation. With the polarized pumping, the lasing threshold will decrease and the slope efficiency of backward output lasing will significantly reduce. Also, the different SOP of the pump resulting in the differences of lasing power properties, including lasing threshold, output power and slope efficiency. But it should be noted that, under the polarized pumping, the linearity of the measured pump side output power vs pump power is not very good. It is 
mainly because the DOP fluctuation when the pump power increases (see the insert in Fig.5 and Fig.6), the DOP value varies from $80 \%$ to $90 \%$ in the high pump side DOP case and varies from $20 \%$ to $30 \%$ in the low pump side DOP case. The different DOP value of the different pump power can have the influence on the Raman gain value. However, in the simulation, the Raman gain is treated as an invariant in each case.

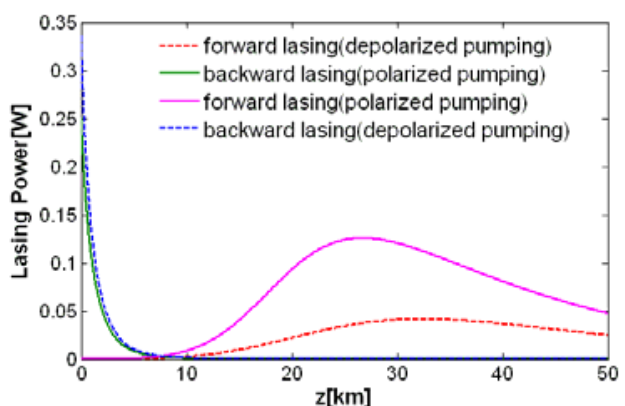

Fig.8 Power distribution with the polarized and depolarized pumping

TABLE II

The values of $g_{f}$ and $g_{b}$ for three cases.

\begin{tabular}{|c|c|c|}
\hline & $g_{f}\left(\mathbf{W}^{-1} \mathbf{k m}^{-1}\right)$ & $g_{\boldsymbol{b}}\left(\mathbf{W}^{-1} \mathbf{k m}^{-\mathbf{1}}\right)$ \\
\hline $\begin{array}{c}\text { Depolarized } \\
\text { pumping }\end{array}$ & 0.50 & 0.50 \\
\hline High DOP & 0.69 & 0.53 \\
\hline Low DOP & 0.62 & 0.51 \\
\hline
\end{tabular}

\section{Conclusions}

In conclusion, we have studied the polarization properties of random fiber laser via Raman gain and Rayleigh feedback utilizing polarized pump. The generated random lasing is partially polarized, the SOP of the output lasing is kept as a stable point on the Poincare sphere. The random lasing' spectrum shows the discrete narrow spectral features with the polarized pumping. Also, the lasing threshold, lasing spectral shape, output power, SOP and DOP all vary substantially with different SOP of pumping. The measured output power at the pump side and fiber far-end indicate that, with the polarized pumping, due to the different average gain coefficient for forward and backward Stokes waves, the random lasing's power distribution is changed and the slope efficiency of pump side output is significantly reduced comparing to the depolarized pumping. Furthermore, considering the dependence of Raman gain on the polarization, we use the modified power balance model by changing the gain factor of forward and backward Stokes waves to qualitatively study the impact of polarization evolution on lasing power properties. The numerical results can explain the experimental results to some extent. We believe that our work has improved the understanding of the polarization optics of random lasing in fibers and formed a base to establish the vector model of random fiber lasers.

\section{Acknowledgments}

This work is supported by ERC Ultralaser project, Natural Science Foundation of China (61205048, 61290312), Research Fund for the Doctoral Program of Higher Education of China (20120185120003), Fundamental Research Funds for the Central Universities (ZYGX2012J002), the PCSIRT project (IRT1218), and the 111 project (B14039).

\section{References}

[1] D. S. Wiersma (2008). The physics and applications of random lasers. Nat. Phys., 4(5), 359-367.

[2] H. C. Hsu, C. Y. Wu, and W. F. Hsieh (2005). Stimulated emission and lasing of random-growth oriented $\mathrm{ZnO}$ nanowires. J. Appl. Phys., 97, 064315.

[3] S. V. Frolov, M. Shkunov, A. Fujii, K. Yoshino, and Z. V. Vardeny (2000). Lasing and stimulated emission in $\pi$-conjugated polymers. IEEE $J$. Quantum Electron., 36(1), 2-11.

[4] R. C. Polson and Z. V. Vardeny (2004). Random lasing in human tissues. Appl. Phys. Lett., 85(7), 1289-1291.

[5] H. Cao, Y. G. Zhao, S. T. Ho, E. W. Seelig, Q. H. Wang, and R. P. H. Chang (1999). Random laser action in semiconductor powder, Phys. Rev. Lett.. 82(11), 2278-2281.

[6] B. Redding, M. A. Choma, and H. Cao (2012). Speckle-free laser imaging using random laser illumination. Nat. Photonics, 6(6), 355-359.

[7] Z. N. Wang, Y. J. Rao, H. Wu, P. Y. Li, Y. Jiang, X. H. Jia, and W. L. Zhang (2012). Long-distance fiber-optic point-sensing systems based on random fiber lasers. Opt. Express, 20, 17695-17700.

[8] X. H. Jia, Y. J. Rao, F. Peng, Z. N. Wang, W. L. Zhang, H. J. Wu, and Y. Jiang (2013). Random-lasing-based distributed fiber-optic amplification. Opt. Express, 21, 6572-6577. 
[9] X. H.Jia, Y. J.Rao, C. X. Yuan, J. Li, X. D. Yan, Z. N. Wang, W. L. Zhang, H. Wu, Y. Y. Zhu, and F. Peng (2013) Hybrid distributed Raman amplification combining random fiber laser based 2nd-order and low-noise LD based 1st-order pumping. Opt. Express, 21, 24611-24619.

[10] O. Frazao, C. Correia, J. L. Santos, and J. M. Baptista (2009). Raman fibre Bragg-grating laser sensor with cooperative Rayleigh scattering for strain-temperature measurement. Meas. Sci. Technol., 20(4), 045203.

[11] J. Nuño, M. Alcon-Camas, and J. D. Ania-Castañón (2012). RIN transfer in random distributed feedback fiber lasers. Opt. Express, 20, 27376-27381.

[12] S. K. Turitsyn, S. A. Babin, A. E. El-Taher, P.Harper, D. V. Churkin, S. I. Kablukov, J. D. Ania-Castañón, V.Karalekas, and E. V. Podivilov (2010). Random distributed feedback fiber laser. Nat. Photonics, 4, 231-235.

[13] S. K. Turitsyn, S. A. Babin, D. V. Churkin, I. D. Vatnik, M. Nikulin, E. V. Podivilov (2014). Random distributed feedback fibre lasers. Physics Reports, in press.

[14] S. A. Babin, A. E. El-Taher, P. Harper, E. V. Podivilov, and S. K. Turitsyn (2011). Tunable random fiber laser. Phys. Rev. A, 84, 021805(R).

[15] A.M. R. Pinto, O. Frazão, J. L. Santos, and M. Lopez-Amo (2010). Multiwavelength fiber laser based on a photonic crystal fiber loop mirror with cooperative Rayleigh scattering. Appl. Phys. B, 99, 391-395.

[16]A. E. El-Taher, P. Harper, S. A. Babin, J. D. Ania-Castanon, D. V. Churkin, E. V. Podvilov, S. K. Turitsyn (2011). Effect of Rayleigh-scattering distributed feedback on multiwavelength Raman fiber laser generation. Opt. Lett., 36, 130-132.

[17] H. Wu, Z. N. Wang, X. H. Jia, P. Y. Li, M. Q. Fan, Y. Li, and Y. Y. Zhu (2013). Flat amplitude multiwavelength Brillouin-Raman random fiber laser with a half-open cavity. Appl. Phys. B, 112(4), 467-471.

[18] S. Sugavanam, Z. Yan, V. Kamynin, A. S. Kurkov, L. Zhang, and D. V. Churkin (2014). Multiwavelength generation in a random distributed feedback fiber laser using an all fiber Lyot filter. Opt. Express, 22, 2839-2844.

[19] S. Sugavanam, N. Tarasov, X. Shu, and D. V. Churkin (2013). Narrow-band generation in random distributed feedback fiber laser. Opt. Express, 21(14), 16466-16472.

[20] I. D. Vatnik, D. V. Churkin, S. A. Babin, and S. K. Turitsyn (2011). Cascaded random distributed feedback Raman fiber laser operating at $1.2 \mu \mathrm{m}$. Opt. Express, 19, 18486-18494.
[21]Z. N. Wang, H. Wu, M. Q. Fan, Y. J. Rao, X. H. Jia, and W. L. Zhang (2013). Third-order random lasing via Raman gain and Rayleigh feedback within a half-open cavity. Opt. Express, 21, 20090-20095.

[22] W. L. Zhang, Y. J. Rao, Z. X. Yang, Z. N. Wang, X. H. Jia (2012). Low threshold 2nd-order Random lasing of a fiber laser with a half-opened cavity. Optics Express, 20, 14400-14405.

[23] I. D. Vatnik, D. V. Churkin, and (2012). Power optimization of random distributed feedback fiber lasers. Opt. Express, 20, 28033-28038.

[24] I. D. Vatnik, D. V. Churkin, E. V. Podivilov, and S. A. Babin (2014). High-efficiency generation in a short random fiber laser. Laser Physics Letters, 11(7), 075101

[25] H. Zhang, P. Zhou, H. Xiao, and X. Xu (2014). Efficient Raman fiber laser based on random Rayleigh distributed feedback with record high power. Laser Physics Letters, 11(7), 075104.

[26] Xiaohua H. Wu, Alexey Yamilov, Heeso Noh, Hui Cao, Eric W. Seelig, and Robert P. H. Chang (2004). Random lasing in closely packed resonant scatterers. J. Opt. Soc. Am. B, 21, 159-167.

[27] Sebastian Knitter, Michael Kues, and Carsten Fallnich (2012). Emission polarization of random lasers in organic dye solutions. Opt. Lett., 37, 3621-3623.

[28] Sebastian Knitter, Michael Kues, Michael Haidl, and Carsten Fallnich (2013). Linearly polarized emission from random lasers with anisotropically amplifying media. Opt. Express 21, 31591-31603.

[29] Fengfeng Yao, Wenlong Zhou, Huanting Bian, Yu Zhang, Yanbo Pei, Xiudong Sun, and Zhiwei Lv (2013). Polarization and polarization control of random lasers from dye-doped nematic liquid crystals. Opt. Lett. 38, 1557-1559.

[30] R. H. Stolen (1979). Polarization effects in fiber Raman and Brillouin lasers. IEEE $J$. Quantum Electron. 15,1157-1160.

[31] A. Doutte, P. Suret, and S. Randoux (2003). Influence of light polarization on dynamics of continuous-wave-pumped Raman fiber lasers. Opt. Lett. 28,2464-2466.

[32] Qiang Lin and Govind P. Agrawal (2003). Vector theory of stimulated Raman scattering and its application to fiber-based Raman amplifiers. $J$. Opt. Soc. Am. B 20, 1616-1631.

[33] D. V. Churkin, S. A. Babin, A. E. El-Taher, P. Harper, S. I. Kablukov, V. Karalekas,J. D. Ania-Castañón, E. V. Podivilov, and S. K. Turitsyn (2010). Raman fiber lasers with a random 
distributed feedback based on Rayleigh scattering.

Phys. Rev. A 82(3), 033828. 